Rev. salud pública. 14 sup (2): 32-45, 2012

\title{
Programa madre canguro: una alternativa sencilla y costo eficaz para la protección de los recién nacidos prematuros o con bajo peso al nacer
}

The kangaroo mothers' programme: a simple and cost-effective alternative for protecting the premature newborn or low-birth-weight babies

\author{
Jenny P. Lizarazo-Medina ${ }^{1}$, Juan M. Ospina-Diaz ${ }^{2}$ y Nelly E. Ariza-Riaño ${ }^{3}$
}

1 Hospital San Rafael de Tunja, Universidad Pedagógica y Tecnológica de Colombia. Tunja, Colombia. lizarazojp@yahoo.com

2 Escuela de Medicina, Universidad Pedagógica y Tecnológica de Colombia. Tunja, Colombia. juan. ospina@uptc.edu.co

3 Escuela de Enfermería, Universidad Pedagógica y Tecnológica de Colombia. Tunja, Colombia. nearjuan@yahoo.com

Recibido 25 Mayo 2011/Enviado para Modificación 8 Agosto 2011/Aceptado 3 Noviembre 2011

\section{RESUMEN}

Objetivo Describir la eficacia y logros del PMC sobre la salud y el desarrollo de los recién nacidos prematuros o con bajo peso al nacer en el Hospital San Rafael de Tunja durante el periodo comprendido entre Noviembre de 2007 y Octubre 31 de 2009 en sus diferentes componentes.

Métodos Estudio observacional descriptivo de una cohorte. Se incluyeron 374 recién nacidos prematuros o con bajo peso al nacer, para evaluar factores sociodemográficos del hogar, antecedentes maternos y obstétricos, características y complicaciones del parto y seguimiento hasta las 40 semanas de edad postconcepcional.

Resultados Altas prevalencias de embarazo adolescente $(17,5 \%)$ y en mayores de 35 años (12,6 \%); embarazo no deseado (40,6 \%); baja calidad y disponibilidad de alimentos en la familia; complicaciones como toxemia gravídica, infecciones y ruptura prematura de membranas; peso promedio al nacer de 1969 gramos; peso promedio al egreso 2 742,9 gramos; ganancia promedio de peso por día de 22 gramos.

Conclusiones Se encuentra que la metodología del programa Madre Canguro mejora sustancialmente los aspectos psicológicos de la madre y el pronóstico de salud de los recién nacidos y permite la estabilización de la temperatura corporal, del ritmo de ganancia de peso, a la vez que disminuye los riesgos de complicaciones y de infección nosocomial. También disminuye los costos de atención y estancia hospitalaria. 
Palabras Clave: Nacimiento prematuro, peso al nacer, bienestar del lactante, desarrollo de programas (fuente: DeCS, BIREME).

\section{ABSTRACT}

Objective Describing the efficacy and achievements of the kangaroo mothers' programme (KMP) regarding preterm or low-birth-weight babies' health and development in Hospital San Rafael in Tunja from November 2007 to December 2009.

Methods This was a retrospective observational cohort study; 374 infants born prematurely or having low-birth-weight were included to assess household sociodemographic factors, maternal and obstetric history, delivery characteristics and complications and follow-up until 40 weeks post-conception age.

Results There was a high prevalence of teenage pregnancy $(17.5 \%)$ and in women older than 35 years (12.6\%), unwanted pregnancy (40.6\%), low quality and poor availability of food in families, complications such as preeclampsia, infection and premature rupture of membranes, 1,969 grams average birth weight, 2,742.9 grams average weight on discharge and 22 grams average weight gain per day.

Conclusions It was found that KMP methodology substantially improved the mothers' psychological aspects and health status and the newborns' prognosis and led to stabilising body temperature and weight gain rate while decreasing risks of complications and nosocomial infection. It also lowered health care costs and shortened hospital stay.

Key Words: Prematurity, low birth weight, kangaroo mothers' programme (source: $\mathrm{MeSH}, \mathrm{NLM}$ ).

$\mathrm{E}$ 1 gobierno de Colombia expidió en 2006 la ley 1098, por medio de la cual se promulga el Código de la Infancia y la Adolescencia. En esta norma se proclama el reconocimiento pleno de derechos a los menores de edad, en un contexto integral que inicia en el momento de la concepción y abarca cuidado, protección, alimentación nutritiva y equilibrada, acceso a los servicios de salud, educación, vestuario adecuado, recreación y vivienda segura dotada de servicios públicos esenciales en un ambiente sano, según se define en el Artículo 17.

También en el Artículo 27 se especifica que todos los niños, niñas y adolescentes tienen derecho a la salud integral, a la que se define como un estado de bienestar físico, síquico y fisiológico y no solo la ausencia de enfermedad. En este sentido, ningún Hospital, Clínica, Centro de Salud y demás entidades dedicadas a la prestación del servicio de salud, sean públicas o privadas, podrán abstenerse de atender a un niño, niña 
que requiera de atención en salud. En relación con los niños, niñas y adolescentes que no figuren como beneficiarios en el régimen contributivo o en el régimen subsidiado, el costo de tales servicios estará a cargo de la Nación (1).

De la misma manera, el Decreto 3039 de Agosto de 2007, establece en el Anexo técnico, Objetivo 1 del Plan Nacional de Salud: “...g. Implementar en todas las entidades promotoras de salud-EPS e instituciones prestadoras de servicios de salud-IPS estrategias para mejorar la prevención y control de la retinopatía perinatal, la sífilis gestacional y congénita; la detección y control del hipotiroidismo congénito. h. Impulsar del desarrollo del programa canguro para la atención del recién nacido de bajo peso al nacer en las instituciones prestadoras de servicios de salud, con los criterios de calidad requeridos."

La condición de los recién nacidos prematuros y/o con bajo peso al nacer constituye un enorme reto para los prestadores de servicios de salud, habida cuenta de la complejidad de procedimientos necesarios para lograr un aumento de peso armónico, regulación térmica y metabólica que garanticen un rápido progreso hacia la situación de normalidad fisiológica, propósitos de particular importancia en la atención de los recién nacidos con bajo peso, si se tienen en cuenta los riesgos que de este desequilibrio se desprenden hacia el desarrollo futuro de los niños (2).

El Programa Madre Canguro (PMC) ha sido reconocido como una metodología eficaz y costo efectiva que permite a las instituciones de salud realizar un proceso de cuidado y seguimiento de los recién nacidos prematuros o con bajo peso al nacer, que desde Colombia se ha proyectado al mundo científico y ha consolidado unas técnicas y procedimientos propios, que han mejorado sustancialmente el pronóstico y las secuelas de la condición de prematurez.

El PMC, empezó a funcionar en 1978, en el Instituto Materno Infantil de Bogotá, a cargo de los médicos Edgar Rey S. y Héctor Martínez G. La capacitación de las madres para el cuidado de sus bebés fue una preocupación constante. Todos los días se realizaban charlas con las madres sobre lactancia, nutrición, estimulación y prevención de enfermedades. La invitación se extendía a los padres y a cualquier familiar que quisiera asistir. La capacitación transmitía confianza y cariño a las madres, agregando una condición que se hizo indispensable para su posterior desarrollo: la calidez humana, el buen trato, la solidaridad frente a la difícil situación que enfrentaba la mayor parte de las madres. La doctora Natalie Charpak, en compañía de Martha Girón, 
trabajadora social, Martha Cristo, sicóloga, y Yolanda Cifuentes emprendieron la realización del primer trabajo de investigación sobre Programa Madre Canguro en Colombia entre 1992 y 1993, incluyendo en la muestra población del Instituto Materno Infantil y el Instituto de los Seguros Sociales $(3,4)$.

Según la OMS, en el mundo, cada año cerca de 20 millones de niños nacen con bajo peso, el $90 \%$ de estos nacimientos ocurre en países en vía de desarrollo. En nuestro país, de los 697.029 niños que nacieron en el 2003, el $7,73 \%$ registraron bajo peso al nacer (5), más recientemente se ha reportado una prevalencia del $11 \%$ (3). Diversos factores se han asociado con el bajo peso al nacer y la prematurez, entre ellos destacan: corto intervalo entre embarazos, raza (6), enfermedad periodontal, ocupación y edad de la madre, lugar de residencia, estrato socioeconómico, apoyo familiar, tabaquismo en la madre, deficiencia en el control prenatal (7).

La mortalidad neonatal en Colombia representa proporcionalmente el 57 $\%$ de todas las muertes en el primer año de vida. El departamento de Boyacá, muestra cifras preocupantes, para el Sector Salud, sobre todo si se tiene en cuenta que en la actualidad la mortalidad perinatal y neonatal son indicadores del grado de desarrollo socioeconómico y de bienestar de una población y de las condiciones de salud materna. De la calidad de atención preconcepcional, prenatal, intraparto y del neonato e involucrando también el entorno familiar, depende el desarrollo de una país o de una región. En Colombia la tasa de mortalidad perinatal es de 12 por mil y en Boyacá de 33 por 1000 nacidos vivos (8).

La estrategia del Programa lleva a que la madre canguro desarrolle mayor competencia para cuidar y criar a los bebés prematuros, en comparación con el personal de salud, las madres se hacen más sensibles a las necesidades de salud y desarrollo de los bebés incidiendo en la disminución del índice de morbilidad que presentan estos niños, además se presenta un cambio en la estructura familiar al permitir una mayor participación del padre en el cuidado del hijo y al mejorar la autoestima de la mamá (9).

El PMC se estructura con una metodología específica que busca el logro de los siguientes objetivos concretos:

- Mejorar el pronóstico y los resultados de los niños prematuros y/o con bajo peso al nacer, superando el concepto de «sobrevida» por el de «calidad de vida». 
- Estimular una relación temprana y estrecha entre el binomio madrehijo para desarrollar un adecuado vínculo emocional.

- Humanizar el cuidado hospitalario y ambulatorio de estos bebés.

- Educar y animar madres y familias en el manejo del prematuro y/o bebés con bajo peso al nacer.

- Disminuir el abandono de estos bebés.

- Usar racionalmente la tecnología y reducir costos de hospitalización (10).

El Programa Madre Canguro, establecido en el Hospital San Rafael de Tunja desde el 1 de noviembre del 2007 propende por brindar apoyo a las madres mediante una capacitación que busca proporcionar bases conceptuales y prácticas que lleven a un correcto cuidado de sus hijos en el hogar; de este modo se busca mejorar las condiciones ambientales de desarrollo y maduración para el recién nacido. El presente estudio se propuso describir la eficacia y logros del PMC en el HSRT durante el periodo comprendido entre Noviembre de 2007 y Diciembre de 2009 en sus diferentes componentes.

\section{METODOLOGIA}

Se adelantó un estudio longitudinal de cohorte en el que se incluyeron 374 recién nacidos reclutados en el Programa madre Canguro del Hospital San Rafael de Tunja entre el 30 de Noviembre de 2007 y el 31 de Octubre de 2009. La información fue consignada en un Software facilitado por la Fundación Canguro-Universidad Javeriana, de Bogotá D.C.

Se incluyeron recién nacidos diagnosticados como prematuros o con bajo peso al nacer, cuyas madres aceptaron voluntariamente participar en el programa; para cada uno de ellos se recogió información relevante sobre aspectos demográficos y socioculturales, antropometría y estado clínico, se registró en el seguimiento la alimentación y complicaciones. Posteriormente se evaluó el estado clínico general y antropometría al cumplir cuarenta semanas después de la fecha estimada de la concepción y se evaluaron las diferencias.

Tanto las madres de los bebés inscritos como sus familiares copartícipes en el programa recibieron capacitación sobre la naturaleza y características de las técnicas básicas del PMC, en los momentos intrahospitalario y 
ambulatorio; en particular sobre lo referente a la posición que debe adoptar el bebé de forma permanente (posición vertical de cúbito prono -como una rana-), con sólo una camisa sin mangas y el pañal durante las 24 horas del día para así tener un continuo contacto piel a piel; ésta posición debe mantenerse hasta que el recién nacido muestre signos de regulación térmica, momento en que está indicada su salida de la posición. También se les capacitó sobre la alimentación que debe recibir el prematuro, que consiste en brindar principalmente leche materna, y, si es necesario, utilizar leche complementaria; y sobre cómo extraer y suministrar leche a los recién nacidos (11). En la mayoría de los casos la intervención educativa se adelantó en varios etapas: Actividades grupales de capacitación sobre conceptos teóricos, demostraciones prácticas en talleres participativos; entrevistas semiestructuradas, detección de vacíos conceptuales, se realizó un acompañamiento con intervenciones personalizadas centradas en motivación, reforzamiento y reentrenamiento en los componentes teóricos y prácticos del PMC.

El análisis de datos se llevó a cabo en el Programa Epi-Info ${ }^{\mathrm{TM}}$. Se estimaron porcentajes para las variables discretas con los correspondientes intervalos de confianza al $95 \%$, medias y desviación estándar para las variables continúas. Como variables de interés se incluyó el peso en gramos, la talla en $\mathrm{cm}$, perímetro cefálico en $\mathrm{cm}$, edad gestacional al momento del parto y antropometría al momento de cumplir cuarenta semanas postconcepcionales.

\section{RESULTADOS}

Ámbito Socioeconómico y cultural: La edad promedio del grupo de madres fue 25,9 años $(\mathrm{SD}=8,19) ; 65$ (17,5\%), eran menores de 18 años y $47(12,6$ $\%)$, mayores de 35 años. Noventa y dos madres (24,7 \%), no contaban con pareja estable, aunque el 13,7 \%, contaba con algún tipo de apoyo familiar. $4,1 \%$, de las madres y 5,1\%, de los padres eran analfabetos. 264 madres (71 $\%$ ), tienen por ocupación las actividades de ama de casa y 7,3 \%, estaban desempleadas, mientras que el $4,3 \%$, de los padres no tenían empleo. En lo que se refiere a la calidad de la nutrición en la familia, apenas $8,9 \%$, la considera buena, mientras que un porcentaje similar $8,4 \%$, la considera severamente insuficiente, para el $28,9 \%$, es apenas suficiente y para $47,3 \%$, es insuficiente. En ingreso familiar promedio mensual ascendió a \$ 501984 ( $\mathrm{SD}=550$ 000), unos US\$271, el cual debe alcanzar para la manutención de 
4,3 personas en promedio por cada familia $(\mathrm{SD}=1,7)$; es importante anotar que apenas $25 \%$, de las familias tiene ingresos mensuales superiores a 500 000 pesos mensuales $42,6 \%$, de las familias no cuenta con agua potable en la casa $61,1 \%$, de las madres debía desplazarse durante 1 hora o más tiempo para llegar al Hospital.

Antecedentes maternos y obstétricos: 3,2\%, de las maternas reportaron ser fumadoras y $51(13,7 \%)$, consumían alcohol con frecuencia de ocasional a frecuente. El promedio de embarazos por materna fue 2,45 ( $\mathrm{SD}=5,26), 30,5 \%$, de las mujeres tenían 3 o más embarazos incluyendo el actual. El promedio de controles prenatales fue 5,4 ( $\mathrm{SD}=2,3)$, apenas 6 maternas $(1,6 \%)$, no tuvieron controles. Se detectaron antes del parto 2 casos de Lúes de 72 serologías practicadas, se reportaron además 2 casos de Malaria tratados. Se practicaron 285 análisis de orina, de los cuales 138 (39,1\%), fueron positivos.

Ciento cincuenta y uno $(40,6 \%)$ de los embarazos fueron no deseados y 99 (27\%), ocurrieron aún con el empleo de métodos anticonceptivos, principalmente hormonales $(14,9 \%)$, DIU $(7,3 \%)$, de barrera o locales $(8,4$ $\%)$ y calendario $(3,7 \%)$.

En cuanto a las patologías durante el embarazo, noventa y siete mujeres (26,1 $\%)$, presentaron cuadros de Toxemia Gravídica. Cuarenta y tres embarazos (11,6\%), eran múltiples. Ciento cinco mujeres de 280 analizadas presentaban anemia $(37,5 \%)$. Hubo 68 casos de Amenaza de parto pre-término (18,3\%).

Sufrimiento fetal: Se reportó frecuencia cardiaca fetal anormal en 52 casos $(13,9 \%)$ y Monitoreo anormal en 67 (18 \%). En 34 casos se encontró líquido amniótico meconiado $(9,1 \%)$ y Ruptura prematura de membranas en 106 $(28,4 \%)$.

Parto y adaptación neonatal: 197 (52 \%), de los 374 productos fueron de sexo femenino. El parto vía vaginal ocurrió en 193 maternas $(51,7 \%)$; por cesárea en 179 (48,0 \%), de las cuales solo 23 (6,2 \%), fueron electivas. La escala de Apgar al minuto del nacimiento fue igual o inferior a tres en 4 casos $(1,1 \%)$, de cuatro a siete en 86 casos $(23,0 \%)$, e igual o superior a ocho en 223 casos (59,7 \%); es importante anotar que en 16,1\% de los nacimientos se omitió anotar este dato. El Apgar promedio al minuto del nacimiento fue de 7,7 $(\mathrm{SD}=1,23)$. En 36 casos $(9,7 \%)$, hubo necesidad de maniobras de reanimación. Es importante anotar que solo un bebé registró a los 5 minutos Apgar igual o 
inferior a 4, por cuanto este es un criterio estandarizado de asfixia, que define el pronóstico del niño.

A los cinco minutos del nacimiento el APGAR promedio fue 9,09 $(\mathrm{SD}=1,07)$; igual o inferior a tres en un caso $(0,3 \%)$, entre cuatro y siete en 22 casos $(5,9 \%)$, e igual o superior a ocho en $292(48,4 \%)$, no aparece reporte en 58 casos $(15,5 \%)$.

El peso promedio al momento del nacimiento fue de 1969 gramos $(\mathrm{SD}=401,25)$, no se observaron diferencias estadísticamente significativas por sexo. Los pesos por rango se detallan en la Tabla 1.

Edad gestacional al momento del nacimiento: el promedio de edad gestacional al momento del parto fue de 35,3 semanas ( $\mathrm{SD}=2,7)$. La distribución de edades por sexo se muestra en la Tabla 2.

Tabla 1. Peso de los Recién Nacidos según sexo Programa Madre Canguro Hospital san Rafael de Tunja 2007-2009

\begin{tabular}{lccc}
\hline Peso en gramos & Femenino (\%) & Masculino (\%) & Total $(\%)$ \\
\hline Menos de 1 000 & $4(2,1)$ & $3(1,7)$ & $7(1,9)$ \\
1000 a 1 500 & $24(12,4)$ & $19(10,6)$ & $43(11,6)$ \\
1501 a 2 000 & $64(33,2)$ & $62(34,6)$ & $126(33,9)$ \\
2 001 a 2 500 & $99(51,3)$ & $91(50,8)$ & $190(51,1)$ \\
Más de 2 500 & $2(1,0)$ & $4(2,2)$ & $6(1,6)$ \\
Total & 193 & 179 & 372 \\
\hline
\end{tabular}

Tabla 2. Distribución de la edad gestacional al momento del parto Programa Madre Canguro. Hospital San Rafael Tunja 2007-2009

\begin{tabular}{lcc}
\hline Edad gestacional en semanas & Frecuencia & Porcentaje \\
\hline Menos de 30 (prematuro extremo) & 16 & 4,30 \\
30 a 34 (prematuro) & 104 & 27,90 \\
35 a 37 (prematuro tardío) & 176 & 47,20 \\
38 a 40 (a término) & 77 & 20,60 \\
Total & 373 & 100,00 \\
\hline
\end{tabular}

Patología neonatal: 166 niños requirieron hospitalización en la UCI, se registró un tiempo promedio de estancia de 8,1 días $(\mathrm{SD}=12,4)$; de ellos, 88 (53\%), permanecieron tres días o menos, 37 (33,3\%), entre 4 y 10 días y el 24,7\%, restante más de 10 días. Trecientos seis niños fueron internados en la Unidad Neonatal, para efectos de cuidado intermedio y básico, con una estancia promedio de 17,7 días $(\mathrm{SD}=15,9)$; 11 niños $(3,6 \%)$, durante tres días o menos, 123 (40,2\%), entre cuatro y diez días y el restante 52,2 $\%$, más de 10 días. 
Los diagnósticos específicos de complicación neonatal se consignan en la Tabla 3.

Tabla 3. Diagnósticos específicos de complicación neonatal

\begin{tabular}{lcc} 
Programa Madre Canguro. Hospital San Rafael de Tunja 2007-2009 \\
\hline Diagnóstico & Frecuencia & Porcentaje \\
\hline Ictericia patológica & 155 & 41,4 \\
Oxigeno dependencia & 136 & 36,3 \\
Síndrome dificultad respiratoria & 109 & 29,14 \\
Infección neonatal temprana & 50 & 13,36 \\
Apnea secundaria & 43 & 11,5 \\
Hipoglucemia sintomática & 27 & 7,22 \\
Tono muscular anormal & 15 & 4,0 \\
Hemorragia intraventricular & 11 & 2,9 \\
Infección neonatal tardía & 10 & 2,67 \\
Infección nosocomial & 9 & 2,4 \\
Apnea primaria & 7 & 1,87 \\
Convulsión & 6 & 1,6 \\
\hline
\end{tabular}

Alimentación: La frecuencia de tipo de alimentación al momento del reclutamiento y al de cumplir cuarenta semanas de edad postconcepcional se muestran en la Tabla 4.

Tabla 4. Tipo de alimentación que recibe el bebé al reclutamiento y a las 40 semanas de edad postconcepcional. Programa Madre Canguro Hospital san Rafael de Tunja 2007-2009

\begin{tabular}{lcccc}
\hline \multirow{2}{*}{ TIPO } & \multicolumn{2}{c}{ Reclutamiento } & \multicolumn{2}{c}{40 semanas } \\
\cline { 2 - 5 } & Frecuencia & Porcentaje & Frecuencia & Porcentaje \\
\hline Leche humana & 188 & 53,7 & 256 & 82,6 \\
Leche humana y artificial & 125 & 35,7 & 50 & 16,1 \\
Leche artificial & 5 & 1,4 & 11 & 3,5 \\
\hline
\end{tabular}

El promedio de edad en días desde el nacimiento hasta completar 40 semanas de edad postconcepcional fue de 34,6 días $(\mathrm{SD}=17,9)$; el promedio de peso al completar cuarenta semanas de edad postconcepcional fue de 2742,9 gramos ( $\mathrm{SD}=439,9)$. De esta manera se registra una ganancia promedio de peso en este período de 786,0 gramos $(\mathrm{SD}=494,6)$, lo que significaría una ganancia neta promedio de 11,2 gramos por kilogramo de peso y por día.

Se registraron, hasta el momento de cumplir las cuarenta semanas postconcepcionales, 37 readmisiones, principalmente por falla en el crecimiento (14), alteración de los reflejos o del tono muscular (11), 
retinopatía del prematuro (4) y enfermedad infecciosa (4).

Mortalidad. Ningún bebé falleció antes de cumplir las 40 semanas de edad postconcepcional ajustada; cuatro de las madres fallecieron antes del egreso del niño.

\section{DISCUSIÓN}

El bajo peso al nacer es una condición clínica que significa un riesgo sustancialmente elevado de morir, hasta 14 veces mayor para los bebés. Los factores generales que se encuentran asociados a él, además de la pobreza, son el bajo nivel de escolaridad, embarazo adolescente, malnutrición de la madre, tabaquismo, presencia de hipertensión arterial durante el embarazo, embarazo múltiple, anemia de la madre, antecedente de aborto inducido, infecciones cérvico-vaginales, incompetencia ístmico-cervical. También se ha reportado que a medida que aumenta la edad materna el peso de los recién nacidos tiende a disminuir (12). A este respecto es importante anotar que la identificación temprana y tratamiento de complicaciones durante el embarazo como las vaginosis y también la administración de calcio suplementario, hierro y folato se identifican como intervenciones oportunas y capaces de disminuir sustancialmente el riesgo de partos pre término o recién nacidos con bajo peso al nacer.

Se ha demostrado la existencia de una fuerte asociación de la condición de bajo peso al nacer con la ocurrencia más frecuente durante los primeros 12 meses de vida, complicaciones severas, entre los que destacan retraso del desarrollo neurológico, hemorragia cerebral, alteraciones respiratorias y otras que imponen la necesidad de atención prolongada y hospitalización. También existe sólida evidencia que prueba la asociación entre el bajo peso al nacer y mayor mortalidad infantil. Estudios epidemiológicos más recientes muestran una posible asociación entre el BPN y enfermedades del adulto tales como la hipertensión arterial y la diabetes mellitus no insulinodependiente (13).

Los resultados del presente estudio son similares a lo reportado en México (14), España (15) y Cali, Colombia (3), con experiencias de seguimiento a recién nacidos con bajo peso y/o prematuros, que demuestran que el cambio en el manejo de los bebés, centrado en la educación y participación activa a las madres sobre la manera de sostener, cuidar y alimentar al niño mejora sustancialmente el pronóstico y la sobrevida (16). La eficacia de la 
técnica asistencial y participativa ha significado un cambio tan significativo sobre la morbilidad y mortalidad que la UNICEF ha promovido el programa a nivel mundial como un método altamente apropiado para proteger la integridad de los recién nacidos (17-18).

Dentro de los alcances más relevantes obtenidos mediante la metodología Madre canguro se han destacado la ganancia de peso a un ritmo uniforme y sostenido, la mejor calidad de atención y cuidado proporcionada al recién nacido, el fortalecimiento de la relación madre-hijo y la reducción significativa en los costos de la atención demostrable en la reducción de la mortalidad, la incidencia de complicaciones severas, infecciones nosocomiales, adecuación de la lactancia materna exclusiva y reducción de la disconformidad materna (19).

Un aspecto relevante de los beneficios del PMC tiene que ver con el fortalecimiento de la relación madre - hijo, en particular con el fortalecimiento de la seguridad y confianza de las mujeres madres de bebés prematuro o con bajo peso al nacer, ya que permite manifestar las preocupaciones referidas al estado de salud y adquirir respuestas concretas acerca de la manera de ayudar en el cuidado de su hijo, lo que influye favorablemente sobre el estado de ansiedad (20).

Los postulados teóricos sobre el concepto de apego, afirman que el comportamiento de las madres constituye un aspecto determinante en la estructuración de las relaciones afectivas primarias del recién nacido, y en estos términos hay algunas variables que inciden mayormente, por ejemplo la sensibilidad de la madre, en la medida en que ella modifica su comportamiento para adecuarlo a las necesidades del niño, para brindarle protección y generar la percepción de seguridad, determinante a su vez del desarrollo sicomotor. El uso de lenguaje verbal también ha sido calificado como prioritario en el afianzamiento del niño (21).

El cuidado del bebé en el domicilio constituye un desarrollo tecnológico en el que se busca que padres y agentes de salud compartan responsabilidades de asistencia y cuidado esenciales en niños cuyo estado de salud es frágil pero estable, y no significa una descarga total del Sistema de Salud respecto de su obligación institucional (22), por cuanto la estrategia central es la humanización de la atención, en la medida en que los profesionales responsables requieren de una capacitación exhaustiva en la comprensión y 
manejo de los problemas psicológicos y prácticos que se pueden derivar de una metodología en la que el contacto piel a piel, la alimentación asistida y el fortalecimiento del apego son garantía del adecuado desarrollo y crecimiento del niño (23).

Desde la perspectiva costo beneficio es importante tener en cuenta que el volumen de demanda de atención especializada para recién nacidos que presentan estado de prematurez y/o bajo peso al nacer está aumentando paulatinamente en los diferentes escenarios de atención materno infantil del país, con mucho mayor intensidad en los ambientes en los que se conjuga además la pobreza e insuficiente accesibilidad a los servicios de alta complejidad, circunstancia que en cierta medida impele a la adopción de metodologías como la estructurada a partir del PMC (24).

El análisis de costo y efectividad en la aplicación del PMC ha demostrado un alivio sustancial para los menguados recursos de las instituciones sanitarias. En el estudio Cattaneo, adelantado en 1998, los costos totales fueron casi $50 \%$, más bajos con el método madre canguro. De la misma manera, en el estudio Sloan de 1994, los costos de la atención neonatal fueron más altos en el grupo control que en el grupo método madre canguro (19). Claro está, esto no significa que sea una estrategia sin costo, pues lo que se ha podido evidenciar es la necesidad de contar con recurso humano altamente calificado, que pueda suplir las necesidades de acompañamiento y monitoreo domiciliario a las madres y familiares comprometidos en el PMC.

Si bien hay un amplio debate en torno a los beneficios de alimentación con leche materna exclusiva como fuente nutricia para los recién nacidos, las dificultades derivadas de la prematurez en torno a la capacidad de succionar o de la madre, en caso de complicaciones, en este y otros estudios se encuentra que la alternación de leche materna con suplementos de leche artificial, constituyen en estas circunstancias, una alternativa válida para garantizar la supervivencia y el desarrollo de los niños (25).

Desde la perspectiva de estabilización clínica, el contacto piel a piel y la alimentación regularizada constituyen herramientas valiosas que influyen sobre la frecuencia de ocurrencia de alteración de la regulación térmica y la presentación de episodios de hipoglicemia o alteraciones de la frecuencia respiratoria, en la medida que el ritmo metabólico y de gasto calórico puede 
permanecer estable por períodos prolongados de tiempo (26).

Se considera que una Infección Nosocomial es aquella documentada clínicamente después de 48 horas de estancia en el hospital y que no estaba presente al ingreso.Una tasa de infección nosocomial de $2,4 \%$, como la encontrada en el presente estudio, es sustancialmente menor a las reportadas en los servicios de hospitalización pediátrica convencional, por ejemplo la reportada en 21 hospitales de México que fue de 9,8\% (27), mientras que la reportada en una unidad de cuidado intensivo neonatal del mismo país ascendió a 35,8 \%, (28). Los hallazgos del análisis descriptivo de nuestra cohorte permiten corroborar en buena parte la existencia de beneficios reales sobre la salud y desarrollo de los neonatos prematuros o con bajo peso al nacer reportada por Bárcenas en 2003 (10): estabilización de los signos vitales, disminución de las apneas y consumo sostenido y equilibrado de Oxígeno, con grados estables y crecientes de saturación de oxígeno, riesgo disminuido de infección nosocomial y mejor pronóstico en las infecciones neonatales tempranas, menor gasto energético y ganancia de peso sostenida, estabilidad de la temperatura corporal, con menos riesgo de hipotermia e hipoglucemia, incremento y sostenimiento de la alimentación con leche materna y mejor vínculo afectivo de la madre con el niño

Agradecimientos: Los autores expresan su gratitud a la Fundación Canguro de Bogotá D.C. y a la Universidad Javeriana, a las directivas del Hospital San Rafael de Tunja, a las madres y familiares, por los aportes en tecnología y logística que permitieron el ensamble y seguimiento de esta cohorte.

\section{REFERENCIAS}

1. Ley 1098 de 2006. República de Colombia. Diario Oficial 46446:1-28

2. Santos D, Assis AM, Bastos AC, Santos LM, Santos CA, Strina A, Prado MS. Determinants of cognitive function in childhood: A cohort study in a middle income context. BMC Public health.2008; 8(1):202-16. [Internet]. Disponible en: http://www.biomedcentral. com/1471-2458/ 8/202. Consultado Octubre 2010.

3. Torres J, Palencia D, Sánchez DM, García J, Rey H, Echandía CA. Programa Madre Canguro: Primeros resultados de una cohorte de niños seguidos desde la unidad neonatal hasta la semana 40 de edad postconcepcional. Colombia Médica 2006; 37(2):96-101.

4. Instituto Materno Infantil. Bogotá. Colombia. Programa Madre Canguro. Historia del programa madre canguro. [Internet]. Disponible en: http://madrecanguro.tripod.com/historia.htm. Consultado Abril 2009.

5. OMS. Sistema de Información Estadística (WHOSIS). [Internet]. Disponible en: http:// www. who.int/whosis/en. Consultado Octubre 2010.

6. Rawlings J, Rawlings V, Read J. Prevalence of low birth weight and preterm delivery in relation to the interval between pregnancies among white and black women. NEJM.1995; 332(2):69-74.

7. Bener A, Abdulrazzaq, Dawodu A. Sociodemographic risk factors associated with low birth 
weight in United Arab Emirates. Journal of Biosocial Science1996; 28:339-346.

8. PROFAMILIA. Colombia. Encuesta Nacional de Demografía y Salud. Profamilia; 2005. [Internet]. Disponible en: www.profamilia.org.co/encuestas/00resumen/011general. htm. Consultado Junio 2009.

9. Charpak N, Figueroa Z. Método madre canguro, guías de manejo. Fundación Canguro. [Internet]. Disponible en: http://fundacioncanguro.co/descargas/reglas- kmcespanol.pdf. Consultado Octubre 2010.

10. Bárcenas G. Programa madre canguro: Medicina basada en la evidencia. Rev Fac Med Univ Nac Colomb.2003; 51(2):80-86.

11. Charpak N, Figueroa Z, Hamel A. El Método Canguro. Padres y Familiares de niños prematuros pueden reemplazar las incubadoras. McGraw Hill. Bogotá; 1997.

12. Peraza G, Pérez SC, Figueroa Z. Factores asociados al bajo peso al nacer. Rev Cubana Med Gen Integr.2001; 17(5):490-496.

13. Lopes A, Port FK. The low birth weight hypothesis as a plausible explanation for the blackwhite differences in hypertension, non-insulin-dependent diabetes and end-stage renal disease. Am J Kidney Dis.1995; 25(2):350-356.

14. Garzón E, Ramírez J. Echeverría A. Experiencias con la técnica Madre Canguro en un hospital de segundo nivel de atención. Rev Mex Pediatría 1999; 66(5):193-196.

15. Closa R, Moralejo J, Ravés MM, Martínez MJ, Gómez A. Método Canguro en recién nacidos prematuros ingresados en una Unidad de Cuidados Intensivos Neonatal. Anales españoles de Pediatría.1998; 49 (5):495-98.

16. Whitelaw A, Liestol K. Mortality an growth of low birth weight infants in the Kangaroo Mother Program in Bogota, Colombia. Pediatrics.1994; 94(6):931-932.

17. Urquizo R. Manejo del Prematuro. Lecturas de Lactancia Materna, Ablactancia y Vacunación Antitetánica. PROCAME II (Lima); 1994.

18. UNICEF. 2009. Las madres canguro de Ghana. [Internet]. Disponible en: http://www.unicef. org/spanish/sowc09/docs/OWC09-Panel-3.5-ESP.pdf. Consultado en Octubre 2010

19. Conde-Agudelo A, Diaz-Rossello JL, Belizan JM. Método madre canguro para reducir la morbimortalidad en neonatos con bajo peso al nacer. Biblioteca Cochrane plus. 2008. $\mathrm{N}^{\circ}$ 2. [Internet]. Disponible en: http://apps.who.int/rhl//reviews/CD002771sp. pdf. Consultado en Octubre 2010.

20. Jarret MH. Parent partners: A parent to parent support program in the NICU. Part II: Program implementation. Pediatr-Nurs, 1996; 14(2):149.

21. Ortiz JA, Borré A, Carrillo S, Gutierrez G. Relación de apego en madres adolescentes y sus bebés canguro. Rev Lat Psicol.2006; 38(1):71-86.

22.Silva KL, Sena R, Leite JCA, Seixas CT, Gonçalves AM. Internação domiciliar no Sistema Único de Saúde. Rev Saúde Publica.2005; 39(3):391-7.

23. Furlan C, Scochi C, Furtado M. Percepção dos pais sobre a vivência no método mãecanguru. Rev Latino-Am. Enfermagem, 2003; 11(4):444-452.

24. Ruiz JG, Charpak N. Guías de práctica clínica basadas en evidencia para la óptima utilización del método madre canguro en el recién nacido pretérmino y/o de bajo peso al nacer. Fundación Canguro y Departamento de Epidemiología y Estadística, Universidad Javeriana; Bogotá, Colombia; 2007.

25. Charpak, N. Ruiz, G. Figueroa, Z. Influence of feeding patterns and other factors on early somatic growth of healthy, preterm infants in home based Kangaroo mother care: a cohort study. Journal of pediatric gastroenterology and nutrition, 2005; 41(3):7.

26. De leeuw R, Colín EM, Dunnebier EZ, Mirmiran M. Physiologic effects of kangaroo care in very small preterm infants. Biol Neonate, 1991; 59:49-55.

27. Avila-Figueroa C. Prevalencia de infecciones nosocomiales en niños: Encuesta de 21 hospitales en México. Salud pública Méx.1999;41(suppl.1):S18-S25

28. Medina M, Hernández I, Nandí ME, Avila C. Infecciones nosocomiales en una unidad de cuidado intensivo neonatal. Perinatol reprod hum, 2000; 14(3):143-150. 